\title{
Rituximab induction therapy for de novo ANCA associated vasculitis in pregnancy: a case report
}

\author{
Claire Harris ${ }^{1}$, Judith Marin ${ }^{2}$ and Monica C. Beaulieu ${ }^{1,2,3^{*}}$
}

\begin{abstract}
Background: The diagnosis of antineutrophil cytoplasmic antibody (ANCA) associated vasculitis (AAV) is rare in pregnancy but potentially life threatening. There are no randomized controlled trials to guide the management of AAV in pregnancy and fetal safety data remains limited. Rituximab administration, a treatment for AAV, has been reported in pregnant women with reassuring fetal outcomes in the oncology and rheumatology literature; however, no published reports describe its use in AAV.

Case presentation: We present a case of de novo myeloperoxidase positive (MPO) AAV diagnosed at 22 weeks gestation. Clinical presentation included elevated serum creatinine at $177 \mu \mathrm{mol} / \mathrm{L}$, hematuria and nephrotic range proteinuria along with high-titre MPO. Diagnosis was confirmed by renal biopsy. Patient was treated with methylprednisolone IV followed by oral prednisone $70 \mathrm{mg}$ daily and Rituximab $650 \mathrm{mg}$ IV weekly for four weeks followed by azathioprine maintenance therapy and prednisone taper. Delivery occurred at 29 weeks gestation via cesarean section for maternal neurologic symptoms concerning for preeclampsia. Maternal and fetal CD 19 cells were depleted at time of delivery with associated fetal lymphopenia in the absence of infection or other complications related to Rituximab use. The patient experienced a reduction in proteinuria and inflammatory markers following Rituximab therapy; however, serum creatinine increased to $375 \mu \mathrm{mol} / \mathrm{L}$ by 11 weeks post-partum.
\end{abstract}

Conclusion: We report the first use, to our knowledge, of Rituximab with corticosteroids for induction therapy of AAV in pregnancy.

Keywords: Antineutrophil cytoplasmic antibody (ANCA), Pregnancy, Case report, Rituximab, Myeloperoxidase (MPO), Vasculitis

\section{Background}

The de novo diagnosis of antineutrophil cytoplasmic antibody (ANCA) associated vasculitis (AAV) is rare in pregnancy. Treatment of AAV in pregnancy poses a therapeutic dilemma as the risks of teratogenicity and adverse fetal outcomes are weighed against the potential fatal maternal outcomes of untreated severe disease. We report the first use, to our knowledge, of Rituximab with corticosteroids for induction therapy in MPO associated ANCA vasculitis with severe renal involvement diagnosed in the second trimester of pregnancy.

\footnotetext{
* Correspondence: MBeaulieu@providencehealth.bc.ca

'Division of Nephrology, University of British Columbia, Vancouver, BC, Canada

${ }^{2}$ St. Paul's Hospital, 1081 Burrard St. Vancouver BC, Vancouver, Canada Full list of author information is available at the end of the article
}

\section{Case presentation}

A 20 year old gravida 1, para 0, female of Salvadoran descent was referred to nephrology for deteriorating kidney function and the nephrotic syndrome at 22 weeks gestational age (GA). Past medical history included a diagnosis of juvenile rheumatoid arthritis at age 12 treated with nonsteroidal anti-inflammatory drugs (NSAIDs) with resolution of symptoms into adulthood without therapy. The patient also endorsed pre-conception use of intranasal cocaine and cigarettes with no use (documented on urine drug screen) post conception. Medication use included prenatal vitamins, diclectin and acetaminophen. The patient reported a family history of rheumatoid arthritis diagnosed in her mother and maternal grandmother.

At presentation the patient's serum creatinine was $177 \mu \mathrm{mol} / \mathrm{L}$ compared to a previous creatinine of $82 \mu \mathrm{mol} /$

(c) The Author(s). 2018 Open Access This article is distributed under the terms of the Creative Commons Attribution 4.0 International License (http://creativecommons.org/licenses/by/4.0/), which permits unrestricted use, distribution, and 
L three months earlier and a pre-pregnancy value of $56 \mu \mathrm{mol} / \mathrm{L}$. She endorsed progressive lower limb edema beginning in early pregnancy. A history of tea coloured urine, epistaxis and sinus pressure was elicited. She did not have hemoptysis or rash but endorsed arthralgias in her shoulders, wrists, distal interphalangeal joints and ankles.

On examination her blood pressure was 116/60 mmHg. Relevant examination findings included pitting edema to the knees bilaterally. There were no active joints or rash.

24-h urine for protein yielded $9.81 \mathrm{~g}$ with $>100 \mathrm{RBC} / \mathrm{hpf}$ seen on microscopy. Urine albumin to creatinine ratio was $450 \mathrm{mg} / \mathrm{mmol}$. Urine drug screen was negative for cocaine. Serum albumin was $20 \mathrm{~g} / \mathrm{L}$, antinuclear antibody 1:80, double stranded DNA negative, extractable nuclear antigen negative, C3, C4, normal and rheumatoid factor, cyclic citrullinated peptide negative, $\mathrm{C}$-reactive protein 13 (normal < 10). HIV, Hepatitis B and C serology were negative. ANCA serology was positive with MPO antibody tire of 107.7 Antibody Index (AI) (normal < 20).

Renal ultrasound revealed structurally normal kidneys. Obstetrical ultrasound revealed mild intrauterine growth restriction (IUGR). Chest x-ray was unremarkable.

Renal biopsy was performed urgently with a diagnosis of pauci immune glomerulonephritis. Three of 14 glomeruli on light microscopy contained cellular crescents, 4 glomeruli fibrous crescents, 1 globally sclerosed. Up to six glomeruli contained segmental sclerosis with only five to $10 \%$ interstitial fibrosis and tubular atrophy.

The patient was counselled regarding the maternal and fetal risks associated with her diagnosis and her therapeutic options including termination and elected to continue with the pregnancy. In particular, the risk of progression of renal impairment and potential requirement of dialysis during pregnancy were discussed, in addition to the high risk of preeclampsia and preterm labour if the pregnancy was continued. The patient was treated with methylprednisolone $1 \mathrm{~g}$ IV daily $\times 3$ followed by oral prednisone $70 \mathrm{mg}$ daily dosed by ideal weight and then Rituximab $650 \mathrm{mg}$ IV weekly for four weeks. No infusion reactions to Rituximab were noted. The patient developed diffuse striae distensae which may have been a result of the high dose prednisone in the context of pregnancy and weight gain. Serum creatinine continued to rise throughout pregnancy to $250 \mu \mathrm{mol} / \mathrm{L}$, despite a reduction in proteinuria to a urine albumin creatinine ratio of $125 \mathrm{mg} / \mathrm{mmol}$ and improvement in symptoms. At 27 weeks GA, the patient became hypertensive necessitating use of labetalol therapy. She was initiated on erythropoietin for anemia. The patient developed thrombocytopenia which was felt to be immune mediated in nature with a nadir of 64 . Repeat obstetrical ultrasounds revealed normalization of fetal growth.

Delivery occurred at 29 weeks GA via caesarean section for worsening maternal headache and visual disturbance concerning for preeclampsia and worsening renal function with serum creatinine of $275 \mu \mathrm{mol} / \mathrm{L}$. Maternal CD19+ cells were depleted at time of delivery. Neonatal birth weight was $1010 \mathrm{~g}$ and the neonate spent 10 weeks in the neonatal intensive care unit before discharge in healthy condition. Neonatal CD19+ cell levels were depleted at birth with associated transient lymphopenia without infectious complications. After delivery, the patient's creatinine rose to a value of $375 \mu \mathrm{mol} / \mathrm{L}$ (eGFR $14 \mathrm{ml} / \mathrm{min}$ ) at 11 weeks post partum. Prednisone was tapered slowly to $10 \mathrm{mg}$ daily and the patient was initiated on azathioprine maintenance therapy four months after Rituximab induction and titrated up to a dose of $2 \mathrm{mg} / \mathrm{kg} /$ day. At the time of maintenance therapy initiation, the MPO titer was 5.8 Units (normal $<1$ ).

\section{Discussion}

Without specific treatment ANCA associated vasculitis is associated with high mortality and poor renal outcomes. The most recent KDIGO guidelines suggest the use of cyclophosphamide and corticosteroids as induction treatment with grade 1A evidence [1]. Rituximab and corticosteroids may be used as an alternative initial treatment in patients without severe disease or in whom cyclophosphamide is contraindicated (1B) [1]. The evidence for treatment of ANCA associated vasculitis in pregnancy is limited to case report and case series data.

Renal dysfunction is a well-established risk factor for pregnancy complications including preeclampsia, preterm labour and pregnancy loss [2]. It is generally accepted that active autoimmune disease such as vasculitis in pregnancy further increase these risks but the literature is limited to case reports in this area and thus attributable risk is unknown [3].

The literature describing ANCA associated vasculitis in pregnancy is heterogeneous and is dominated by descriptions of patients with known ANCA vasculitides in remission prior to conception. In one case study, Presta et al. reported the pre-conception use of plasma exchange, cyclophosphamide and prednisone for MPO-ANCA vasculitis 1 month prior to unplanned conception and there was no fetal complications [4].

De novo ANCA associated vasculitis in pregnancy is rare. A case series of 12 patients with de novo ANCA associated vasculitis in pregnancy was reported by Alfhaily et al. in 2009 [5]. Treatment regimens included combinations of prednisone plus cyclophosphamide, Intravenous immunoglobulin (IVIG) or azathioprine. One fetal death was reported, 2 pregnancies were terminated for medical indications with a mean live birth gestational age of 34 weeks and good fetal outcomes. One patient developed pre-eclampsia. There was one maternal death in this series with the majority of women achieving clinical remission. 
Rituximab is listed as a Food and Drug Administration (FDA) category $\mathrm{C}$ drug per the previous FDA drug classification system [6]. As an IgG isotype antibody, it crosses the placenta beginning at 16 weeks gestation [7]. Pregnancy outcomes after maternal exposure to rituximab have been described in primarily in the oncology literature with some reports of use in autoimmune diseases including rheumatoid arthritis and systemic lupus erythematosus. A review of Rituximab exposure during 9 pregnancies for oncology (6 lymphoma) and autoimmune (1 idiopathic thrombocytopenic purpura, 1 thrombotic thrombocytopenic purpura, 1 autoimmune hemolytic anemia) indications reported no serious neonatal outcomes [6]. Although B cell depletion occurred in $4 / 5$ neonates tested during the first week of life, recovery of B cell populations occurred by 6 months and no serious infections were reported even in the 2 neonates with low white blood cell counts at birth. A possible explanation for the absence of infections reported thus far in the literature is that neonates depend on maternal IgG for immunity during the first few months of life while B-cell function still developing during this time frame even in unexposed normal neonates [8].

A case series by Chakravarty reviewed 153 pregnancies with maternal exposure to rituximab and 90 live births including 21 pregnancies with antenatal exposure [9]. The rate of preterm labor was $19 \%$, compared to $10-12 \%$ in the general population however this rate is comparable to women with chronic medical diseases. The rate of congenital malformations was the same as the general population. The rate of miscarriage was higher possibly due to frequent testing detecting early pregnancies or again due to maternal comorbidities. Four neonatal infections occurred but no pattern was identified regarding timing of rituximab exposure and no infections occurred in the 7 neonates who developed cytopenias.

With respect to the use of rituximab for AAV prior to conception, Pendergraft et al. reported maternal and fetal outcomes in patients who received rituximab pre conception [10]. This retrospective analysis reported 8 pregnancies in 6 women with vasculitis who received rituximab prior to conception. Mean rituximab exposure was 5.2 months prior to estimated date of confinement (EDC), with 4 of 8 pregnancies conceived within one month of most recent rituximab infusion. Rituximab was discontinued at time of pregnancy diagnosis and patients were maintained on prednisone and/or azathioprine. There was one fetal demise due to a congenital anomaly unrelated to rituximab and maternal disease activity was quiescent in all but one pregnancy where prednisone was up titrated due to respiratory symptoms. At delivery 6 of 8 women had undetectable CD20+ B lymphocytes however 3 of 3 of fetal samples had present CD20+ B lymphocytes. Mean delivery occurred at 38 wks GA for the 7 live births.
Review of the literature reveals one prior case report of the use of rituximab for induction therapy in a case of de novo pauci immune glomerulonephritis which was ANCA negative [11]. The pregnancy was ended with termination and patient was switched to cyclophosphamide after receiving two doses of rituximab in addition to corticosteroids and plasmapheresis, therefore no neonatal outcomes are reported.

As a result of this case, we report the first known use of rituximab for induction therapy of ANCA associated vasculitis during an established pregnancy with continuation of the pregnancy to delivery. Although Rituximab exposure in the second and third trimester appears to cause neonatal B cell depletion this does not appear to be associated with adverse events in the neonate. Rituximab exposure does not appear to increase congenital malformations even when exposure occurs in the first trimester or just prior to conception. Further investigation is still required to determine the adequacy of the immune response to vaccination in the offspring and it is recommended that live vaccines should be avoided during the first 6 months of life if exposure occurred after the first trimester [12].

\section{Conclusion}

Although more evidence is required, Rituximab can be considered for the treatment of ANCA vasculitis during pregnancy. The decision to use any immunosuppressive medication in pregnancy requires careful counselling of the patient regarding the limitations of our knowledge in this area and the possible expected fetal outcomes. However, the importance of adequate treatment of a potentially life threatening maternal condition must be highlighted. Our patient developed preeclampsia and premature delivery even with improvement in markers of her vasculitis with rituximab therapy. This outcome emphasizes the high risk nature of pregnancies in patients with severe renal impairment and systemic inflammatory conditions and the need for specialized obstetrical care from a highly trained interprofessional team.

\section{Abbreviations \\ AAV: ANCA associated vasculitis; Al: Antibody Index; ANCA: Antineutrophil cytoplasmic antibody; EDC: Estimated date of confinement; FDA: Food and Drug Administration; GA: Gestational Age; IUGRs: Mild intrauterine growth restriction; IVIG: Intravenous immunoglobulin; MPO: Myeloperoxidase; NSAIDs: Nonsteroidal anti-inflammatory drugs}

Availability of data and materials

Data sharing is not applicable to this article as no datasets were generated or analyzed for this case report.

Authors' contributions

$\mathrm{CH}, \mathrm{MB}$ and $\mathrm{JM}$ were involved in the index case and literature review. $\mathrm{CH}$ collected the data and drafted the manuscript. MB and JM were involved in revising the manuscript. All authors read and approved the final manuscript. 
Ethics approval and consent to participate

Not applicable.

\section{Consent for publication}

Written informed consent was obtained from the patient for publication of this case report.

\section{Competing interests}

The authors declare that they have no competing interests.

\section{Publisher's Note}

Springer Nature remains neutral with regard to jurisdictional claims in published maps and institutional affiliations.

\section{Author details}

'Division of Nephrology, University of British Columbia, Vancouver, BC, Canada. ${ }^{2}$ St. Paul's Hospital, 1081 Burrard St. Vancouver BC, Vancouver,

Canada. ${ }^{3}$ BC Provincial Renal Agency, Vancouver, BC, Canada.

Received: 11 September 2017 Accepted: 17 June 2018

Published online: 28 June 2018

\section{References}

1. KDIGO. KDIGO Clinical Practice Guideline for Glomerulonephritis. Kidney Int Suppl. 2012;2:209. http://www.kdigo.org/clinical_practice_guidelines/pdf/ KDIGO-GN-Guideline.pdf

2. Zhang JJ, Ma XX, Hao L, Liu LJ, Lv JC, Zhang H. A systematic review and meta-analysis of outcomes of pregnancy in CKD and CKD outcomes in pregnancy. Clin J Am Soc Nephol. 2015;10:1964-78.

3. Doria A, Bajocchi G, Tonon M, Salvarani C. Pre-pregnancy counselling of patients with vasculitis. Rheumatology. 2008:47:iii13-5.

4. Presta P, Presta P, Fuiano G, et al. Successful conception and pregnancy in p-ANCA associated vasculitis in course of treatment with immunosuppressive drugs and renal replacement therapy. Int J Rheum Dis. 2015;18:470-2

5. Alfhaily F, Watts R, Leather A. Wegener's granulomatosis occurring de novo during pregnancy. Clin Exp Rheumatol. 2009;27(Suppl 52):S86-8.

6. Vinet E, Pineau C, Gordon C, Clarke AE. Biologic therapy and pregnancy outcomes in women with rheumatic diseases. Arthritis Rheum (Arthritis Care \& Research). 2009;61(5):587-92.

7. Østensen $M$, Lockshin M, Doria A, et al. Update on safety during pregnancy of biological agents and some immunosuppressive anti-rheumatic drugs. Rheumatology. 2008;47(Suppl 3):iii28-31.

8. Klink DT, van Elburg RM, Schreurs MWJ, van Well GTJ. Rituximab administration in third trimester of pregnancy suppresses neonatal B-cell development. Clin Devel Immunol. 2008;2008:6.

9. Chakravarty EF, Murray ER, Kelman A, Farmer P. Pregnancy outcomes after maternal exposure to rituximab. Blood. 2011;117(5):1499-506.

10. Pendergraft WF, McGrath MM, Murphy AP, et al. Fetal outcomes after rituximab exposure in women with autoimmune vasculitis. Ann Rheum Dis. 2013;72(12):2051-3.

11. Conduit C, Yew S, Jose M, Jayne D, Kirkland G. A case of diagnosis anti-neutrophil cytoplasmic antibody-negative pauci-immune necrotising glomerulonephritis in pregnancy. Intern Med J. 2017;47(5):600-1.

12. Götestam Skorpen C, Hoeltzenbein M, Tincani A, et al. The EULAR points to consider for use of antirheumatic drugs before pregnancy, and during pregnancy and lactation. Ann Rheum Dis. 2016;75:795-810.

\section{Ready to submit your research? Choose BMC and benefit from:}

- fast, convenient online submission

- thorough peer review by experienced researchers in your field

- rapid publication on acceptance

- support for research data, including large and complex data types

- gold Open Access which fosters wider collaboration and increased citations

- maximum visibility for your research: over $100 \mathrm{M}$ website views per year

At BMC, research is always in progress.

Learn more biomedcentral.com/submissions 